\title{
ANOTHER APPROXIMATION THEORETIC CHARACTERIZATION OF INNER PRODUCT SPACES
}

\author{
DAN AMIR AND FRANK DEUTSCH ${ }^{1}$
}

\begin{abstract}
A normed space $E$ is an inner product space if and only if for every 2-dimensional subspace $V$ and every segment $I \subset V$, the corresponding metric projections satisfy the commutative property $P_{I} P_{V}=P_{V} P_{I}$.
\end{abstract}

For a subset $A$ of a normed linear space $E$, we denote by $P_{A}$ the metric projection on $A$, i.e. the set-valued mapping which corresponds to each $x \in E$ the (possibly empty) set of its best approximations in $A: P_{A} x=\{y \in A$; $\|x-y\|=d(x, A)\} . A$ is called proximinal if $P_{A} x$ is nonempty for every $x \in E$. If $E$ is a Hilbert space and $A$ is a closed subspace of $E$, then $P_{A}$ is just the (single-valued) orthogonal projection onto $A$.

There are several known characterizations of inner product spaces which can be stated in terms of the metric projections. See e.g. [3], [5], [8], and [9]. We shall consider three other such conditions below. For all of these three characterizing conditions, the necessity part is immediate. The weakest condition (hence strongest characterization) is due to Lorch [7] (cf. also Day [1, p. 152]):

(L) If $\|x\|=\|y\|=1$ and $A=\{\beta x+y / \beta ; \beta \neq 0$ real $\}$, then $x+y \in$ $P_{A} 0$.

The next condition, due to Gurari and Sozonov [4], is:

(GS) If $\|x\|=\|y\|=1$ and $A$ is the segment $[x, y]=\{\alpha x+(1-\alpha) y$; $0 \leqslant \alpha \leqslant 1\}$, then $(x+y) / 2 \in P_{A} 0$.

The third condition (under the assumption $\operatorname{dim} E \geqslant 3$ ) is due to Joichi [6]:

(J) If $V$ is a 2-dimensional subspace of $E, u \in E$ with $0 \in P_{V} u$ and $A=S_{0}$ is the "unit circle" in $V: S_{0}=\{v \in V:\|v\|=1\}$, then $P_{A} u=A$.

We first give an easy proof that $(\mathrm{J}) \Rightarrow(\mathrm{GS}) \Rightarrow(\mathrm{L})$, and hence this yields an alternate approach to the more involved sufficiency proofs as given in [4] and [6].

(J) $\Rightarrow$ (GS): We may assume $\operatorname{dim} E=3$. If (GS) fails, then there exists $x, y$ in $E$ with $\|x\|=\|y\|=1$ and $0 \leqslant \gamma<\frac{1}{2}$ such that the element $z=\gamma x+(1$ - $\gamma$ )y satisfies $\|z\|=\min _{0 \leqslant \lambda \leqslant 1 / 2}\|\lambda x+(1-\lambda) y\|<\left\|\frac{1}{2}(x+y)\right\|$. Extend the segment $[x, y]$ to a supporting hyperplane $V+z$ to the ball $\{w \in E$ :

Received by the editors July 25, 1977.

AMS (MOS) subject classifications (1970). Primary 46B99; Secondary 41A65.

$K e y$ words and phrases. Inner product space, Hilbert space, metric projection.

'Supported in part by a grant from the National Science Foundation.

(c) American Mathematical Society 1978 
$\|w\| \leqslant\|z\|\}$ at $z$. Let $u=-z /\|x-z\|$ and $v=(x-z) /\|x-z\|$. Then $0 \in P_{V} u$ and $\pm v \in S_{0}$, but

$$
\|u-v\|=\frac{\|x\|}{\|x-z\|} \neq \frac{\|2 z-x\|}{\|x-z\|}=\|u+v\|
$$

(since if $\|x\|=\|2 z-x\|=\|y\|$, then the three distinct collinear points $x, y$, and $2 z-x$ have the same norm so all the points in $[x, y]$ must have the same norm, a contradiction to $\left.\|z\|<\left\|\frac{1}{2}(x+y)\right\|\right)$. Thus (J) fails.

(GS) $\Rightarrow(\mathrm{L})$ : Suppose (GS) holds. If $\|x\|=\|y\|=1, \beta \neq 0$, and $\gamma=\beta / 2$ $+1 / 2 \beta$, then $|\gamma| \geqslant 1$. Hence

$$
\left\|\beta x+\frac{1}{\beta} y\right\| \geqslant 2\left\|\frac{\beta}{2 \gamma} x+\frac{1}{2 \beta \gamma} y\right\| \geqslant 2\left\|\frac{1}{2}(x+y)\right\|=\|x+y\|
$$

(since $\beta / 2 \gamma+1 / 2 \beta \gamma=1$ ). Hence (L) holds.

The characterization we add here is by a commutativity property of the metric projection.

THEOREM. The following are equivalent for a normed space $E$ with $\operatorname{dim} E \geqslant 3$ :

(1) $E$ is an inner product space;

(2) For every proximinal subspace $V$ and any nonempty $A \subset V, P_{A} \circ P_{V}=$ $P_{A}\left(=P_{V} \circ P_{A}\right)$

(3) The same as (2) with $V$ 2-dimensional and $A$ a segment.

Proof. (1) $\Rightarrow(2)$. This is immediate from the orthogonality of $x-P_{V} x$ to $V:$ if $x \in E, y=P_{V} x, z \in P_{A} y$, and $a \in A$, then

$$
\|x-z\|^{2}=\|x-y\|^{2}+\|y-z\|^{2} \leqslant\|x-y\|^{2}+\|y-a\|^{2}=\|x-a\|^{2}
$$

and equality holds if and only if $\|y-a\|=\|y-z\|$, i.e. $a \in P_{A} y$.

$(2) \Rightarrow(3)$ is trivial.

$(3) \Rightarrow(1)$. Since a normed space $E$ is an inner product space iff each 2-dimensional subspace is, we may assume $\operatorname{dim} E=3$.

We show first that $E$ is strictly convex. If not, we can find a 2-dimensional subspace $V$ and $x \in E$ such that $P_{V} x$ is not a singleton. We may assume also that 0 is a boundary point of $P_{V} x$ in $V$. Let $K$ be the cone $\left\{\lambda P_{V} x: \lambda \geqslant 0\right\} . K$ is contained in a halfspace, therefore $K \neq V$ and we can find $v \in V$, $\|v\|=1$, with $0<d(v, K)<\frac{1}{2}$ and $\lambda>0, y \in P_{V} x$ with $\|\lambda y-v\|<\frac{1}{2}$. Let $A=[0, v]$. Since $v \notin K, A \cap P_{V} x=\{0\}$ and $P_{A} x=0$. On the other hand, if $0 \in P_{A} y$, then $\|y\| \leqslant\|y-\gamma v\|$ for all $\gamma \in[0,1]$, hence for all $\gamma \geqslant 0$. It follows that $\|\lambda y\| \leqslant\|\lambda y-\lambda \gamma v\|$ for all $\gamma \geqslant 0$. In particular, $\|\lambda y\| \leqslant\|\lambda y-v\|<\frac{1}{2}$ while $\|\lambda v\| \geqslant\|v\|-\|\lambda y-v\|>\frac{1}{2}$, a contradiction. Thus $E$ is strictly convex.

We shall show that for every 2-dimensional subspace $V$ of $E$ and $x \in E$ with $P_{V} x=0$, the nonempty intersections of "spheres" around $x$ with $V$ are multiples of the unit circle in $V$, i.e. if $y_{0} \in V$ is arbitrary, then $S_{1}=\{v \in V$ : $\left.\|x-v\|=\left\|x-y_{0}\right\|\right\}$ coincides with $\left\|y_{0}\right\| S_{0}=\left\{v \in V:\|v\|=\left\|y_{0}\right\|\right\}$. By $(\mathrm{J})$, this guarantees that $E$ is an inner product space. 
The idea of the proof is to show that $S_{1}$ is a curve "parallel" to $S_{0}$, i.e. that every line supporting one of them is parallel to a line supporting the other at the corresponding point. If not, we can find a line segment $[y, z], y \in S_{1}$, such that $(y, z]$ is contained in one of the domains bounded by $S_{1}$ and $\|y\| S_{0}$ and disjoint to the other, so that $y$ is exactly one of the points $P_{[y, z]} x$ or $P_{[y, z]} 0$. It is very natural to conclude from this that $S_{1}$ and $S_{0}$ are proportional, but the formal proof we have uses differentiability properties of convex functionals.

Let $\rho_{i}$ be the Minkowski functional in $V$ of the convex hull of $S_{i}$, i.e. $\rho_{0}(v)=\|v\|, \rho_{1}(v)=1$ if $v \in S_{1}$, and $\rho_{1}$ is positively homogeneous. Since $E$ is strictly convex, $\rho_{1}$ is well defined. The $\rho_{i}$ are convex and therefore $\Delta_{i}(y, w$; $t)=t^{-1}\left[\rho_{i}(y+t w)-\rho_{i}(y)\right]$ is a nondecreasing function of $t>0$ and $\tau_{i}(y$, $w)=\lim _{t \rightarrow 0^{+}} \Delta_{i}(y, w ; t)$ exists for every $y, w$ in $V$ (see [2, p. 446]). If $\gamma>0$, then

$$
\tau_{i}(\gamma y, w)=\lim _{t \rightarrow 0^{+}} \Delta_{i}(\gamma y, w ; t)=\lim _{t \rightarrow 0^{+}} \Delta_{i}\left(y, w ; \frac{t}{\gamma}\right)=\tau_{i}(y, w) .
$$

Fix $y \in S_{1}$ and $w \in V$ which is not in $\operatorname{span}\{y\}$. Let $\gamma_{1}=1$ and $\gamma_{0}=$ $1 /\|y\|$, so that $\gamma_{i} y \in S_{i}$ and hence $\rho_{i}\left(\gamma_{i} y\right)=1$ for $i=0,1$. Denote $\tau_{i}=\tau_{i}(y$, $w)$. Consider the lines

$$
l_{i}(\beta)=l_{i}(y, w ; \beta)=\left(1-\beta \tau_{i}\right) \gamma_{i} y+\beta w, \quad \beta \text { real. }
$$

If $\beta>0$ is small enough so that $\beta \tau_{i}<1$, we have

$$
\rho_{i}\left(l_{i}(\beta)\right)-1=\beta\left[\Delta_{i}\left(\gamma_{i} y, w ; \frac{\beta}{1-\beta \tau_{i}}\right)-\tau_{i}\right] \geqslant 0 .
$$

If $S_{i}(\beta)=l_{i}(\beta) / \rho_{i}\left(l_{i}(\beta)\right)$ (the radial projection of $l_{i}(\beta)$ onto $\left.S_{i}\right)$, then

$$
\frac{1}{\beta} \rho_{i}\left[l_{i}(\beta)-S_{i}(\beta)\right]=\Delta_{i}\left(\gamma_{i} y, w ; \frac{\beta}{1-\beta \tau_{i}}\right)-\tau_{i} \rightarrow 0 \text { as } \beta \rightarrow 0^{+},
$$

i.e. $\rho_{i}\left(l_{i}(\beta)\right)=1+o(\beta)$ as $\beta \rightarrow 0^{+}$. (This means that $l_{i}(\beta)$ is "tangent" to $S_{i}$ at $\gamma_{i} y$ from the $\beta>0$ direction.) Let

If $\gamma_{i} \tau_{i}<\gamma_{j} \tau_{j}$, then for some $\varepsilon>0, \gamma_{i} \Delta_{i}\left(\gamma_{j} y, w ; t\right)<\gamma_{j} \tau_{j}$ for all $0<t \leqslant \varepsilon$.

$$
z=\frac{1}{\gamma_{j}} l_{j}\left(\frac{\varepsilon}{1+\varepsilon \tau_{j}}\right) \text { and } A=[y, z] .
$$

If $u \in(y, z]$, i.e. $u=l_{j}(\beta) / \gamma_{j}$ for $0<\beta \leqslant \varepsilon /\left(1+\varepsilon \tau_{j}\right)$, then $0<\beta /(1-$ $\left.\beta \tau_{j}\right) \leqslant \varepsilon$ and

$$
\begin{aligned}
\rho_{k}(u)-\rho_{k}(y) & =\rho_{k}\left[\left(1-\beta \tau_{j}\right) y+\beta w / \gamma_{j}\right]-\rho_{k}(y) \\
& =\frac{\beta}{\gamma_{k} \gamma_{j}}\left[\gamma_{k} \Delta_{k}\left(\gamma_{j} y, w ; \beta /\left(1-\beta \tau_{j}\right)\right)-\gamma_{j} \tau_{j}\right]
\end{aligned}
$$

is negative for $k=i$ and nonnegative for $k=j$, i.e. $\rho_{i}(u)<\rho_{i}(y)$ and $\rho_{j}(u) \geqslant \rho_{j}(y)$ for all $u \in(y, z]$. In the case $i=0$, we thus have $\|u\|<\|y\|$ 
and $\|x-u\| \geqslant\|x-y\|$ for all $u \in(y, z]$ so that $P_{A} x=y \neq P_{A} 0=P_{A} P_{V} x$, which contradicts (3). While in the case $i=1$, we have $\|u\| \geqslant\|y\|$ and $\|x-u\|<\|x-y\|$ for all $u \in(y, z]$ so that $P_{A} P_{V} x=P_{A} 0=y \neq P_{A} x$, again contradicting (3). Thus we must have $\gamma_{i} \tau_{i}=\gamma_{j} \tau_{j}$, i.e. $\tau_{0}=\|y\| \tau_{1}$ and therefore $l_{1}(\beta)=\|y\| l_{0}(\beta /\|y\|)$. Since $\rho_{1}\left(l_{1}(\beta)\right)=1+o(\beta)$ and $\rho_{0}\left(l_{0}(\beta /\|y\|)\right)=1+o(\beta)$, we have $\rho_{0}\left(l_{1}(\beta)\right)=\|y\|+o(\beta)$ as $\beta \rightarrow 0^{+}$. Therefore, the function

$$
\psi(\beta)=\frac{\rho_{0}\left(l_{1}(\beta)\right)}{\rho_{1}\left(l_{1}(\beta)\right)}, \quad \beta \geqslant 0,
$$

(which represents the ratio between the radially corresponding points on $S_{0}$ and $S_{1}$ in the one-sided neighborhood of the $y$-direction determined by $w$ ) satisfies

$$
\psi_{+}^{\prime}(0)=\lim _{\beta \rightarrow 0^{+}} \frac{\psi(\beta)-\|y\|}{\beta}=0 .
$$

But the same applies to the derivative of the ratio from the other direction, so that the two-sided derivative of this ratio at $y$ is 0 . Since $y$ was any point on $S_{1}$, the ratio is the constant $\left\|y_{0}\right\|$, i.e. $S_{1}=\left\|y_{0}\right\| S_{0}$.

ADDED IN PROOF. The proof of the implication $(3) \Rightarrow(1)$ can be substantially shortened by eliminating everything that comes after the paragraph which shows $E$ is strictly convex, and substituting the following in its place. Recall Hirschfeld's characterization [3]: If $E$ is a strictly convex nonEuclidean 3-dimensional space, there is a one-dimensional subspace $L$ with $P_{L}$ nonlinear. Let $V$ be any 2-dimensional subspace of $E$ containing $L$. Since metric projections onto maximal subspaces are linear, $P_{V}$ and $P_{L} P_{V}$ are linear, which shows that $P_{L} \neq P_{L} P_{V}$. Take any $x$ with $P_{L} x \neq P_{L} P_{V} x$ and let $A=\left[P_{L} x, P_{L} P_{V} x\right] \subset L$. Then $P_{V} P_{A} x=P_{A} x=P_{L} x \neq P_{L} P_{V} x=P_{A} P_{V} x$.

\section{REFERENCES}

1. M. M. Day, Normed linear spaces, 3rd ed., Springer-Verlag, New York, 1973.

2. N. Dunford and J. T. Schwartz, Linear operators, Volume I, Interscience, New York, 1958.

3. R. A. Hirschfeld, On best approximation in normed vector spaces. II, Nieuw Arch. Wisk. 6 (1958), 99-107.

4. N. I. Gurari and Y. I. Sozonov, On normed spaces which have no bias of the unit sphere, Mat. Zametki 7 (1970), 307-310; English transl., Math. Notes 7 (1970), 187-189.

5. R. C. James, Inner products in normed linear spaces, Bull. Amer. Math. Soc. 53 (1947), $559-566$.

6. J. T. Joichi, More characterizations of inner product spaces, Proc. Amer. Math. Soc. 19 (1968), 1185-1186.

7. E. R. Lorch, On some implications which characterize Hilbert space, Ann. of Math. (2) 49 (1948), 523-532.

8. R. R. Phelps, Convex sets and nearest points, Proc. Amer. Math. Soc. 8 (1957), 790-797.

9. W. Rudin and K. T. Smith, Linearity of best approximation: A characterization of ellipsoids, Proc. Nederl. Akad. Wetensch. Ser. A 64 (1961), 97-103.

Department of Mathematical Sciences, Tel-Aviv University, Tel-Aviv, IsRael

Department of Mathematics, Pennsylvania State University, University Park, PennSYLVANIA 16802 